\title{
CORRECTION TO "CONSTRUCTION METHODS FOR BHASKAR RAO AND RELATED DESIGNS”
}

The above paper, by Peter B. Gibbons and Rudolf Mathon, appeared in Volume 42 (1987), 5-30.

Pages 10 and 11 are misplaced, and should appear between pages 17 and 18 .

In the table for $\operatorname{GBRD}\left(4,3,6 ; Z_{2}\right) C_{2}$ in Appendix A2, the off-diagonal 0 (row 2, column 6) should be -. 